\title{
Rotura espontánea de esófago: un problema diagnóstico en la urgencia
}

\author{
R. LANA SOTO, J. L. MENDOZA HERNÁNDEZ', F. ARRANZ GARCÍA, \\ A. NIETO SÁNCHEZ, C. CUERVO MOLINERO, L. JIMÉNEZ DE DIEGO (+) \\ ${ }^{1}$ Unidad de Aparato Digestivo. Fundación Hospital Alcorcón. Servicio de Urgencias. \\ Hospital Clinico San Carlos. Madrid
}

\author{
SPONTANEOUS PERFORATION OF THE ESOPHAGUS: DIFFICULT \\ DIAGNOSIS ON THE EMERGENCY SERVICE
}

\begin{abstract}
RESUMEN
Presentamos dos casos de rotura espontánea de esófago (síndrome de Boerhave) que acudieron a urgencias por distintos síntomas, en ambos casos el diagnóstico se realizó en una segunda visita tras varias horas de evolución del cuadro. El síndrome de Boerhaave es una entidad grave, infrecuente y de difícil diagnóstico debido a su presentación inespecífica, y en el que es fundamental lograr un diagnóstico y tratamiento precoz por su alta morbimortalidad.
\end{abstract}

PALABRAS CLAVE: Rotura espontánea de esófago. Síndrome de boerhaave.

\begin{abstract}
We present two case of a spontaneous perforation of the esophagus (Boerhaave's syndrome), they were associated with different symptoms. Both of them the diagnosis was make on the second admision to the emergency service. The Boerhaave's syndrome is potentially lethal, rela tively rare and the difficult diagnosis in the initial phases. It's a life thre ating condition demanding early diagnosis and rapid aggresive manage ment to prevent fulminant death.
\end{abstract}

KEY WORDS: Spontaneous perforation of the esophagus. Boerhaave's syndrome.

Lana Soto R, Mendoza Hernández JL, Arranz García F, Nieto Sánchez A, Cuervo Molinero C, Jiménez de Diego L. Rotura espontánea de esófago: un problema diagnóstico en la urgencia. An Med Interna (Madrid) 2003; 20: 88-90.

\section{INTRODUCCIÓN}

La ruptura esofágica espontánea tras vómitos (Síndrome de Boerhaave) es una causa poco frecuente de perforación esofágica siendo habitual la demora en el diagnóstico debido a su presentación inespecífica. Las alteraciones radiológicas secundarias a la perforación esofágica se dan en el $80 \%$ de los casos y suelen aparecer varias horas o días después, esto unido a las manifestaciones clínicas inespecíficas dificulta el diagnóstico inicial. Es un proceso con alta mortalidad y los principales factores pronósticos son el tamaño y localización de la perforación, la contaminación de la pleura y del mediastino y la demora en el diagnóstico.

\section{CASOS APORTADOS}

Caso 1: Varón de 71 años con antecedentes personales de hepatopatía crónica; cardiopatía isquémica, portador de doble stent en coronaria derecha; hernia de hiato y un episodio de hemorragia digestiva alta por antiinflamatorios no esteroideos 2 años antes. Tras tomar un analgésico por dolor articular desarrolla intensa epigastralgia seguida de vómitos alimentarios por lo que acude a urgencias. En la exploración física se objetiva una hernia supraumbilical reducible y no dolorosa. Se realiza un hemograma y una bioquímica sanguínea, una radiografía de tórax y otra de abdomen simple sin alteraciones. El dolor cede con ranitidina por lo que se da de alta al paciente con protectores gástricos para continuar en observación domiciliaria. A las 48 horas acude de nuevo por disnea y dolor torácico. En la exploración física el paciente está taquipneico y sudoroso con hipoventilación del campo pulmonar izquierdo y una saturación de oxígeno del $85 \%$. En la radiografía de tórax se aprecia un derrame pleural muy importante, se coloca en urgencias drenaje endotorácico drenando $1500 \mathrm{cc}$ de líquido achocolatado. En el tomografía computerizada (TC) torácica se observa un hidroneumotorax a tensión con neumomediastino y probable rotura esofágica con fístula esófago-pleural. En quirófano se objetiva una laceración esofágica longitudinal de 7 $\mathrm{cm}$. Se realiza una esofaguectomía subtotal conservando $15 \mathrm{~cm}$ de esófago. Durante el postoperatorio desarrolla un cuadro de disfagia que se resuelve tras dilatación endoscópica. El paciente es dado de alta asintomático.

Trabajo aceptado: 12 de noviembre de 2001

Correspondencia: Raquel LanaSoto. C/ Andrés Mellado, 76 Bis, $3^{\circ}$ H. 28015 Madrid. 
Caso 2: Varón de 81 años con antecedentes personales de cardiopatía isquémica y un episodio de hemorragia digestiva alta por antiinflamatorios no esteroideos 4 años antes. Acude al hospital por un cuadro de pesadez postprandial seguido de vómitos alimentarios y un intenso dolor lumbar tras el esfuerzo. En la exploración física únicamente destaca un dolor selectivo a la palpación de apófisis espinosas a la altura de L1-L2. Se realiza hemograma y bioquímica sanguínea sin alteraciones, radiografía de tórax y dorsolumbar en las que se aprecia osteoartrosis dorsolumbar y aorta abdominal calcificada. El dolor desaparece tras la administración de analgésicos, se comprueba una buena tolerancia oral a líquidos y se envía a su domicilio asintomático y con analgesia oral. A las 12 horas acude de nuevo por disnea de aparición brusca y dolor torácico. En la exploración se aprecia importante enfisema subcutáneo, el paciente impresiona de gravedad, está taquipneico y mal perfundido con hipoventilación del campo pulmonar derecho. En la radiografía se confirma un neumotorax derecho y al colocar el tubo de tórax sale contenido bilioso. Ante la sospecha de ruptura esofágica se hace TC torácica (Figs. 1 y 2): importante enfisema subcutáneo, neumomediastino, derrame pleural bilateral y atelectasia de ambos lóbulos inferiores junto con extravasación del contraste oral sin poder identificar el punto de rotura. En quirófano se realiza gastroscopia: desgarro profundo a nivel de cardias (Mallory Weis) con perforación a ese nivel. Broncoscopia intraoperatoria: broncoaspiración. Durante el acto quirúrgico se localiza el punto de rotura, se extirpa la zona de la perforación y se coloca un tubo de torax en el lado izquierdo. El paciente ingresa en UCI falleciendo a los 5 días por fracaso multiorgánico.

\section{DISCUSIÓN}

Se describen dos casos de rotura espontánea de esófago con diferente forma de presentación y en los que se llegó al diagnóstico de manera diferida. Las causas más comunes de perforación esofágica son las debidas a procedimientos médicos (instrumentación y cirugía), los traumatismos (cerrados, abiertos y barotraumas) y los vómitos forzados (síndrome de Boerhaave) (1). En la actualidad los procedimientos médicos causan más de la mitad de las perforaciones (50-70\%) (2). Las perforaciones esofágicas surgidas extrahospitalariamente, si bien son menos comunes que las que se producen en el ámbito médico y quirúrgico, presentan una mayor morbimortalidad

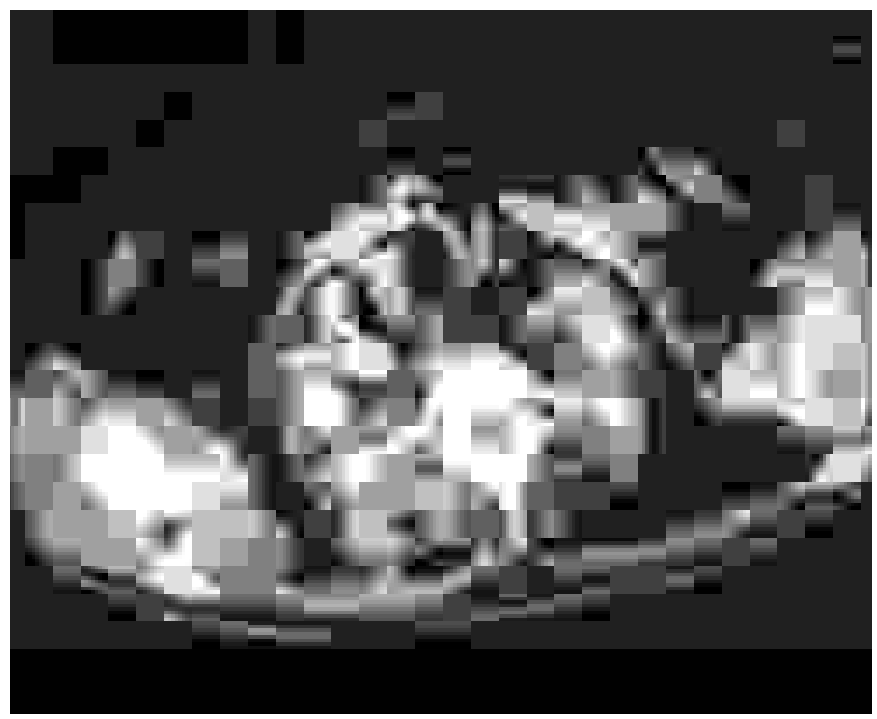

Fig. 1. Tomografía computerizada torácica: corte alto donde se aprecia un gran enfisema subcutáneo.

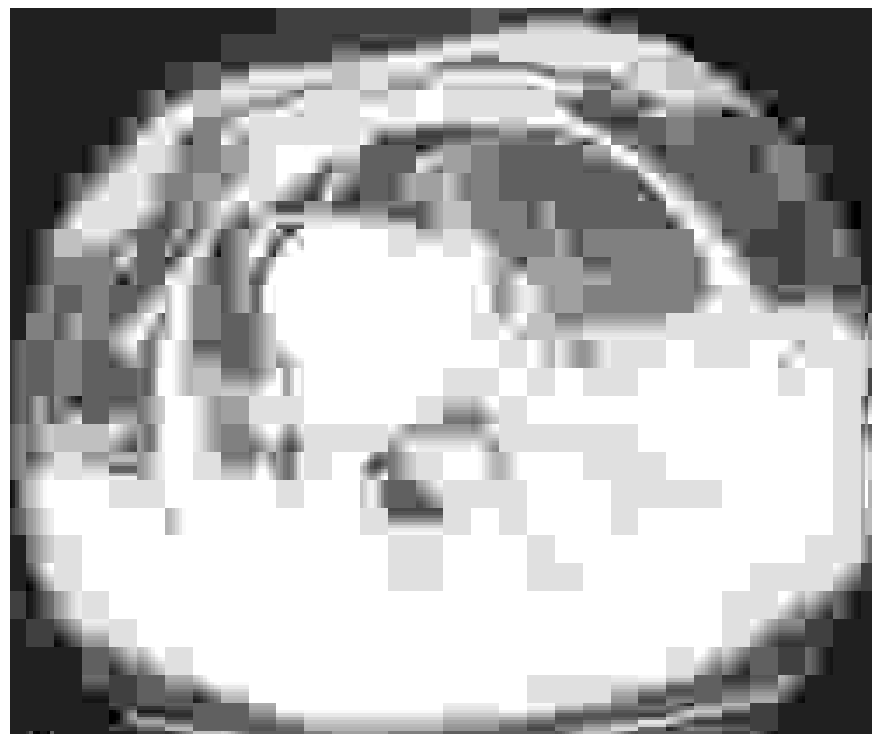

Fig. 2. Tomografía computerizada torácica: enfisema subcutáneo, neumomediastino, neumotórax bilateral, derrame pleural bilateral, atelectasia de ambos lóbulos inferiores con extravasación del contraste oral a espacio pleural..

$(3,4)$. Fundamentalmente por la demora en el diagnóstico, que induce abscesos mediastínicos y pleurales, necrosis tisular y septicemia (5). Entre las perforaciones espontáneas destaca el síndrome de Boerhaave por su rareza, gravedad y difícil diagnóstico debido a la inespecificidad de sus síntomas.

Las perforaciones de esófago habitualmente presentan como síntomas la odinofagia, el dolor torácico, cervical, abdominal y dificultad respiratoria, si bien, prácticamente todas las series publicadas señalan demoras en el diagnóstico, por lo inespecífico de este cuadro. Los síntomas y signos de presentación dependen de la causa, localización y duración de la perforación. El síntoma más común es el dolor torácico retroesternal, que puede empeorar al tragar o respirar, y que no se produjo en nuestros casos. Las rupturas espontáneas de esófago secundarias a accesos violentos de vómitos se presentan habitualmente con dolor abdominal y no torácico. Dada la alta tasa de casos fatales debido fundamentalmente a la demora en el diagnóstico y a su presentación inespecífica se impone pensar en este proceso cuando un sujeto presenta arcadas violentas.

Las radiografías simples muestran anormalidad en más del $80 \%$ de las perforaciones esofágicas. No obstante, pueden ser normales poco después de la perforación, dado que algunas de las complicaciones habituales, como el derrame pleural y el ensanchamiento mediastínico, sólo aparecen después de transcurridos horas o días. Así ocurrió en nuestros casos que tras una presentación atípica con radiografía normal acudieron a urgencias horas después con un cuadro grave y alteraciones radiológicas importantes. Las alteraciones más tardías de colecciones hidroaéreas y abscesos se pueden ver en las radiografías simples, pero se aprecian mejor en la TC. Es preciso practicar estudios radiológicos con contraste del esófago para objetivar de manera completa la localización y el tamaño de la perforación. Se deben utilizar medios de contraste hidrosoluble, en pequeños bolos, y evitar las aspiración debido a la toxicidad de estos agentes. 
Las perforaciones esofágicas tienen una alta mortalidad que varía entre el 6 y el 34\%, y que exige un tratamiento precoz (2). Los determinantes más importantes de supervivencia son el tamaño y la localización de la perforación, la edad avanzada, la contaminación grosera o no del mediastino y las cavidades pleurales, así como el periodo transcurrido entre la perforación y el diagnóstico. Los enfoques no quirúrgicos sólo son aplicables en situaciones muy específicas y la mayoría de los autores recomiendan intervención quirúrgica inmediata en los casos de perforación espontánea. En los últimos tiempos el tratamiento combinado quirúrgico agresivo, junto con los antibióticos y la nutrición parenteral, mejora los resultados en estos pacientes (6). Recientemente se ha comunicado la utilización de endoprótesis autoexpandibles cubiertas como una alternativa terapéutica rápida, segura y efectiva en el tratamiento del síndrome de Boerhaave (7).

Con estos casos pretendemos llamar la atención sobre la necesidad de pensar en la existencia de una perforación espontánea de esófago en pacientes con nauseas violentas, dada la presentación atípica de este proceso y las implicaciones pronósticas de un diagnóstico precoz.

\section{Bibliografía}

1. McDonald GB. Enfermedades esofágicas provocadas por infección, enfermedades sistémicas, fármacos y traumatismos. En: Sleisenger $\mathrm{MH}$, Fordtran JS, eds. Enfermedades gastrointestinales: fisiología, diagnóstico y tratamiento. Buenos Aires: Editorial Médica Panamericana S.A., 1994; 436-63.

2. Solomonov A, Best LA, Goralnik L, Rubin AE, Yigla M. Pleural empyema: An unusual presentation of esophageal perforation. Respiration 1999; 66: 366-8.

3. Jones WG, Ginsberg RJ. A continuing medical challenge. Ann Thorac Surg 1992; 53: 534-43.
4. Handry J, Carolyn ER. Esophageal Injury. En: Baue AE ed. Glenn's Thoracic and Cardiovascular Surgery, ed 6. London, Applenton \& Lange, 1994. p. 615-7.

5. Attar S, Hankins JR, Suter CM. Esophageal perforation. Arch Surg 1991; 78: 1211-3.

6. Reeder LB, DeFilippi VJ, Ferguson MK. Current results of therapy for esophageal perforation 1995; 169: 615-7.

7. Davies AP, Vaughan R. Expanding Mesh Stent in the emergency treatment of Boerhaave's syndrome. Ann Thorac Surg 1999; 67: 1482-3. 